\title{
Mandibular repositioning appliance in the treatment of obstructive sleep apnea syndrome-mini review
}

\begin{abstract}
The sleep is an activity that takes one third of our lives, being fundamental to the maintenance of the mental, physical and emotional health of our organism. The Obstructive Sleep Apnea Syndrome (OSAS) is a respiratory disturb related to the sleep, characterized by the total or parcial obstruction of the superior airways. It is a chronic, progressive and incapacitating disease which is now considered of public health. Dentistry integrates the multidisciplinary team of treatment of the sleep disturbances through, through the mandibular repositioning appliance (MRA). The good acceptability and good results obtained with its use has attracted the attention of sleep specialists, and it has propelled the dentistry to develop not only an ideal apparatus but the capacity to act in an integral way in the treatment of OSAS The objective of this review is to contextualize the mandibular repositioning appliance in the treatment of the OSAS.
\end{abstract}

Keywords: oral appliance, obstructive sleep apnea, mandibular advancement, polysomnography, therapy
Volume 8 Issue 3 - 2017

\author{
Thays Crosara Abrahao Cunha,' Thais De \\ Moura Guimaraes, ${ }^{2}$ Paulo Cesar Simamotto \\ Junior,' Thulio Marquez Cunha, ${ }^{3}$ Lia Rita \\ Azeredo Bittencourt ${ }^{2}$ \\ 'Department of Occlusion, Fixed Prosthesis and Dental \\ Materials, Federal University of Uberlandia, Brazil \\ ${ }^{2}$ Department of Psychobiology, Federal University of Sao Paulo, \\ Brazil \\ ${ }^{3}$ Department of Pneumology, Federal University of Uberlandia, \\ Brazil
}

Correspondence: Thays Crosara Abrahao Cunha, Department of Occlusion, Fixed Prosthesis and Dental Materials, Federal University of Uberlandia, Brazil, Rua Dos Pica Paus, 1750-Acaris 55-CEP, 384 I2-64I, Uberlandia, Brazil,

Email thayscrosara@gmail.com

Received: September 23, 2017 | Published: October 02, 2017
Abbreviations: 0SAS, obstructive sleep apnea syndrome; MRA, mandibular repositionig appliance; CPAP, continuous positive air pressure; TMD, temporomandibular dysfunction.

\section{Introduction}

Obstructive Sleep Apnea Syndrome (OSAS) is a sleep-related respiratory disorder that affects individuals of all ages, from neonates to the elderly. It is characterized by recurrent episodes of total obstruction (apnea) or partial (hypopnea) of the upper airways simultaneously with respiratory effort during sleep leading to intermittent hypoxia and awakenings. ${ }^{1}$ It is a chronic disease, progressive and incapacitating, responsible for high mortality rate and cardiovascular morbidity. Highly prevalent it affects about $30 \%$ of the adult population, being considered a public health problem. ${ }^{2,3}$ Once the diagnosis is adequate and the presence of OSAS is confirmed, treatment should be started as soon as possible, seeking to restore the health and quality of life of this individual. Dentistry integrates the multidisciplinary team of treatment of the sleep disturbances through the mandibular repositioning appliance (MRA). This is a conservative, reversible and cost-effective treatment mode that is quite attractive to the patient. When properly indicated and well executed, MRA provides a treatment that can be considered effective, physiological and comfortable. The good acceptability and good results obtained with its use has attracted the attention of sleep specialists, and it has propelled the dentistry to develop not only an ideal apparatus but the capacity to act in an integral way in the treatment of OSAS. Therefore, it is important that the dentist who is a member of a multiprofessional team focused on their treatment, has knowledge about differential diagnosis, pathophysiology of these disorders, possible treatment options, as well as understanding the mechanisms of action that should guide the indication of MRA.

\section{Obstructive sleep apnea syndrome (OSAS)}

\section{Diagnosis and clinical aspects}

Current diagnostic criteria recommend documentation of the presence of at least one symptom (unintended sleep episodes during wakefulness, excessive daytime sleepiness, not repairing sleep, fatigue or insomnia, waking up with breathing pauses, choking or asphyxia, partner reporting loud snoring and / or breathing pauses during sleep) or Comorbidity (Systemic Arterial Hypertension, Mood Disorder, Cognitive Impairment, Coronary Artery Disease, Vascular Brain Accident, Congestive Heart Failure, Atrial Fibrillation, or Type 2 Diabetes Mellitus) associated with the presence of at least five obstructive respiratory events (apnea, hypopnea and awakening associated with respiratory effort) per hour of sleep (in the registry of polysomnography or ambulatory cardiopulmonary devices). The presence of 15 or more obstructive respiratory events per hour, in the absence of symptoms or comorbidities, is also sufficient for the diagnosis of OSAS. ${ }^{4}$ The severity of the disease is due to the index of respiratory stops that the patient can present. In adults, the occurrence of 5 to 15 respiratory events per hour of sleep classifies the individual as having mild apnea. If this index is between 15 and 30, he is considered to have moderate OSAS. Over 30 respiratory events per hour of sleep make him a severe apneic. ${ }^{4}$ OSAS leads to excessive daytime sleepiness with risk of work accidents, in traffic and has been shown to contribute to the development of systemic arterial hypertension. It is associated with changes in mood, cognition, worsening of quality of life, impaired glucose metabolism and other cardiovascular diseases such as stroke, coronary artery disease, congestive heart failure and atrial fibrillation. ${ }^{5}$ Observational studies point to a higher rate of cardiovascular morbidity and mortality in patients with severe and untreated OSAS. ${ }^{6-8}$ 


\section{Pathophysiology of OSA}

The pathophysiology of OSAS is complex and multifactorial, is a heterogeneous disease, with several contributing factors, besides the individual variability, for the clinical presentation of the patients. The main risk factors are male gender, age progression, obesity and craniofacial structure, and in the latter two factors the genetic and ethnic constitution can play a determining role. ${ }^{5}$ Both the soft tissue of the upper airway and craniofacial characteristics contribute synergistically to the collapse of the pharynx, the so-called "anatomical balance". ${ }^{5-10}$ In addition, other factors in the pathophysiology of OSAS have been blamed for pharyngeal collapse. Among these is the response of the electromyography activity of the dilator muscles of the pharynx,,$^{11-14}$ mechanism of respiratory instability also known as Loop Gain increased (increased minute ventilation response to stimuli such as hypoxemia, hypercapnia, and increased resistance to upper airway)..$^{15,16}$ In addition to these factors, some patients with OSAS may present a decrease in the arousal threshold, which would also lead to hyperventilation, hypocapnia and central and obstructive apnea due to the same reason described above. ${ }^{17,18}$ Corroborating with the pathophysiology of OSAS, recent studies have shown that the displacement of fluid from the lower portion of the body to the cervical region when the patient assumes the decubitus, leads to accumulation of fluid around the pharynx and contributes to the collapse of the pharynx..$^{19,20}$

\section{Craniofacial characteristics of OSAS patients}

Craniofacial structures are considered risk factors for OSAS, as they have an intimate relationship with upper airway patency. The main factors related to the disease are maxillary deficiencies (mainly mandibular), increase of the inferior height of the face, base of the short skull and narrowing of the arch. Not only are the craniofacial bone characteristics responsible for decreasing the upper airway, and increasing the predisposition to OSAS. It is believed that the imbalance between craniofacial phenotype and obesity located near the pharynx is actually a risk factor for disease. Perhaps, a retrognatia associated with the small tongue has the same prediction for OSAS as normal mandible associated with bulky tongue. In relation to soft tissue structures, volumetric increases of the tongue, soft palate and neck are described, with consequent inferior position of the hyoid bone. The craniofacial and soft tissue characteristics should be analyzed, taking into account mainly the balance between them. ${ }^{21}$

\section{Treatments}

Advances in sleep medicine and improved diagnostic tools have led to more effective controls and therapies for this disease. The gold standard of treatment of OSAS continues to be CPAP (Continuous Positive Airway Pressure), while the low adherence to the treatment stimulates the continuous search for alternative methods. Currently, in addition to CPAP, treatment for OSA involves behavioral measures, surgeries, and mandibular repositioning appliances. ${ }^{22}$ Considered a gold standard for OSAS, CPAP consists of the use of a nasal or oronasal mask, attached to equipment that generates pressurized airflow to the upper airflow producing elevation of intraluminal pressure. ${ }^{23-26}$ From a physiological point of view, CPAP acts by providing pneumatic support that prevents the collapse of the upper airway and, consequently, abnormal respiratory events. There is no doubt that the constant nocturnal use of CPAP (At least $5 \mathrm{~h}$ ) produces improvement in the quality of life of patients and, in many cases, of their roommates. ${ }^{23-25}$ It has been shown to reduce excessive daytime sleepiness and cognitive changes associated with OSAS. Most studies also suggest that the adequate use of CPAP reduces blood pressure levels in patients with OSAS with and without systemic arterial hypertension. ${ }^{23}$ However, low acceptance and low adherence to treatment are its main clinical limitations. Sleep hygiene can be described as the practice of behaviors that facilitate and improve sleep quality. Do physical exercises often, maintain regular sleep, and avoid behaviors that interfere with sleep such as smoking, alcohol and caffeine use at night. It is believed that these measures may reduce daytime drowsiness, depression, and increase quality of life. However, there are few studies on the association between sleep hygiene and functional outcomes, especially in patients with OSAS. ${ }^{27}$

\section{Mandibular repositioning appliance (MRA)}

Mandibular advancement appliance has been used as first choice in the treatment of primary snoring and in mild sleep apnea. In patients with moderate and severe OSA, MRAs are indicated for those who did not accept, did not tolerate CPAP or have any contraindications for their use. ${ }^{28}$ Currently there are several models of devices being used for mandibular advancement in the treatment of OSAS. Understanding its mechanism of action to be truly effective is fundamental so that the dental professional knows how to choose the best option for each patient. Good results depend basically on the following factors: material and method used in its manufacture, the type of appliance (monobloc or two plates), and whether it is titrable or fixed. Both the design and the complexity of the mandibular repositioning device influence the efficacy of this therapy.

\section{Indication and contraindication}

According to Guideline published by the American Academy of Sleep Medicine and the American Academy of Sleep Dentistry in 2015 28, MRA is recommended as the first choice when the patient has only primary snoring (absence of OSAS). The same should be prescribed by a sleep doctor for adult patients with OSAS. The dentist must present qualification in sleep; the device must be titled and not pre-fabricated. Still according to the guideline, it is best for the sleep specialist to prescribe MRA rather than prescribe any treatment for patients who have not tolerated CPAP or who prefer an alternative form of treatment. The qualified dentist should follow the treatment in an attempt to reduce the incidence of side effects. The sleep specialist should request polysomnography to evaluate the effectiveness of MRA in follow-up visits and that the patient should make follow-up returns at both the dentist and the sleep physician. ${ }^{28}$

\section{Predictors of success}

Among the main positive predictors described so far are: patients with mild OSAS, women, less obese and younger individuals, as well as those with the majority of apnea events when the subject is in the supine position. ${ }^{29,30}$ Some cephalometric and anatomical parameters have been described in a very controversial way. Dal Fabbro et al. ${ }^{31}$ affirm that the main ones are shorter soft palate, greater retropalatal space, smaller or pharynx, longer maxilla, and shorter hyoid-plane mandibular distance. Almeida et al.$^{32}$ have correlated the MRA titration position during the PSG with the final mandibular position that MRA should achieve for daily use. ${ }^{32}$ This titration consists of a progressive advancement of the mandible guided by the improvement of the symptoms during the PSG and has proved effective in determining if the patient is a possible responder to this therapy. ${ }^{32}$ The same author in 2009 showed that it is possible to optimize MRA results 
by increasing mandibular advancement during a polysomnographic titration in patients with an incomplete response to treatment. ${ }^{33}$ Tsuiki et al. ${ }^{34}$ observed better results with MRA in patients who had required CPAP pressure below $10.5 \mathrm{cmH} 2034$. Despite these several possible predictors of success for MRA in the treatment of OSA already described in the literature, they are still not definitive, since the studies present differences in the criteria of success adopted, in the design of the employed MRA, in the configuration of the samples (age, BMI, cervical circumference, pre-treatment AHI, gender) and in the final mandibular position reached thus affecting the results obtained. ${ }^{35}$ Still other factors such as the activity of the pharyngeal dilator muscles may also be important and likewise the anatomical and functional contributions may differ between patients. It is suggested that mandibular advancement in the treatment of OSAS may be more complex than simply altering the VAS caliber.

\section{Dental protocol}

This therapy requires individual device preparation and a titration period until the jaw reaches the maximum comfortable position, requiring time for both the trained and the dentist. A determining factor in the successful treatment of obstructive sleep apnea with MRA is adherence to treatment, with the most frequent factors of discontinuation being discomfort and reduced or not achieved by treatment. Therefore, correct dressing, good adaptation and effective stability of the appliance to the dental arch are fundamental to the success of this therapy modality. Patients should be submitted to the dental arches molding to make dental models in plaster. A bite record of the mandibular position should be performed using condensing silicone on a record fork (George Gaugeâ) with a value of $50 \%$ of maximal mandibular protrusion. The mandibular advancement should be slow and progressive until it reaches the maximum comfortable protrusion or the total advancement allowed by the expanders. After intervention and progressive adjustment of MRA patients should remain sleeping with the appliance for two months in the position of maximum comfortable mandibular protrusion and then should be referred to the physician for reassessment.

\section{Side effects and complications}

As main factors of nonadherence to MRA, side effects related to Temporomandibular Dysfunction (TMD) and mainly aesthetic / function related effects provided by undesirable dental movements are mentioned. Short-term side effects are dry mouth, excessive salivation, mucosal irritation, morning occlusal alteration, and dental discomfort and TMD pain. They are considered of low degree of discomfort, relatively easy to handle and tend to disappear with good accompaniment. ${ }^{22}$ However, it should be remembered that the discomfort is subjective and it is up to the dental surgeon to manage each patient adequately and individually. Despite the various models of mandibular propellers, all are supported in the dental arches generating vertical and horizontal forces, which can perform undesirable dental movements. The main movements are retroinclination of the upper incisors, vestibuloinclination of the lower incisors, distoinclination of the upper molars, mesioinclination of the lower molars, as well as extrusion of the lower molars. ${ }^{22}$ However, the side effects are minimal compared to the benefits provided by the treatment of the disease. Therefore, tooth movements should be monitored regularly as well as the possible onset of muscle pain. The dentist should be able to diagnose, intervene and minimize these changes before taking treatment for OSA with MRA. It is also extremely important that the patient is aware of these possible side effects and agrees with the treatment. If we consider the severity of OSAS, the potential side effects are outweighed by the benefits generated by the treatment of the disease. Therefore, the treatment should not be interrupted, as long as the dentist is attending the movements and is able to minimize these side effects. It is very important that the patient is aware of the potential side effects of therapy before starting treatment.

\section{Conclusion}

The intraoral mandibular repositioning appliances are effective when well indicated properly made, individualized and aiming for the greatest comfort for the patient. It is imperative that the dentist have knowledge about medicine and sleep biology, so that it can integrate a multidisciplinary team for the treatment of OSAS.

\section{Acknowledgments \\ FOUFU, FAPEMIG, CAPES, CNPq.}

\section{Conflicts of interest}

The authors don't have conflict of interest to disclosures.

\section{Funding}

None.

\section{References}

1. Sleep-related breathing disorders in adults: recommendations for syndrome definition and measurement techniques in clinical research. The Report of an American Academy of Sleep Medicine Task Force Sleep. 1999;22(5):667-689.

2. Tufik S, Santos-Silva R, Taddei JA, Bittencourt LRA. Obstructive sleep apnea syndrome in the Sao Paulo epidemiologic sleep study. Sleep Med. 2010;11(5):441-446.

3. Lavie P, Lavie L, Herer P. All-cause mortality in males with sleep apnoea syndrome: declining mortality rates with age. Eur Respir J. 2005;25(3):514-520.

4. Sateia MJ. International classification of sleep disorders-third edition: highlights and modifications. Chest. 2014;146(5):1387-1394.

5. Jordan AS, McSharry DG, Malhotra A. Adult obstructive sleep apnoea. Lancet. 2014;383(9918):736-747.

6. Campos-Rodriguez F, Martinez-Garcia MA, de la Cruz-Moron I, et al. Cardiovascular mortality in women with obstructive sleep apnea with or without continuous positive airway pressure treatment: a cohort study. Ann Intern Med. 2012;156(2):115-122.

7. Marin JM, Carrizo SJ, Vicente E, et al. Long-term cardiovascular outcomes in men with obstructive sleep apnoea-hypopnoea with or without treatment with continuous positive airway pressure: an observational study. Lancet. 2005;365(9464):1046-1053.

8. Martínez-García MA, Campos-Rodríguez F, Catalán-Serra P, et al. Cardiovascular mortality in obstructive sleep apnea in the elderly: role of long-term continuous positive airway pressure treatment: a prospective observational study. Am J Respir Crit Care Med. 2012;186(9):909-916.

9. Dempsey JA, Veasey SC, Morgan BJ, et al. Pathophysiology of sleep apnea. Physiol Rev. 2010;90(1):47-112.

10. Tsuiki S, Isono S, Ishikawa $\mathrm{T}$, et al. Anatomical balance of the upper airway and obstructive sleep apnea. Anesthesiology. 2008;108(6):1009-1015.

11. Genta PR, Owens RL, Edwards BA, et al. Influence of pharyngeal muscle activity on inspiratory negative effort dependence in the human upper airway. Respir Physiol Neurobiol. 2014;201:55-59. 
12. Gleadhill IC, Schwartz AR, Schubert N, et al. Upper airway collapsibility in snorers and in patients with obstructive hypopnea and apnea. Am Rev Respir Dis. 1991;143(6):1300-1303.

13. Mezzanotte WS, Tangel DJ, White DP. Waking genioglossa electromyogram in sleep apnea patients versus normal controls (a neuromuscular compensatory mechanism). $J$ Clin Invest. 1992;89(5):1571-1579.

14. Mezzanotte WS, Tangel DJ, White DP. Influence of sleep onset on upperairway muscle activity in apnea patients versus normal controls. Am Respir Crit Care Med. 1996;153(6 Pt 1):1880-1887.

15. Wellman A, Jordan AS, Malhotra A, et al. Ventilatory control and airway anatomy in obstructive sleep apnea. Am J Respir Crit Care Med. 2004;170(11):1225-1232.

16. Younes M, Ostrowski M, Thompson W, et al. Chemical control stability in patients with obstructive sleep apnea. Am J Respir Crit Care Med. 2001;163(5):1181-1190.

17. Eckert DJ, Owens RL, Kehlmann GB, et al. Eszopiclone increases the respiratory arousal threshold and lowers the apnoea/hypopnoea index in obstructive sleep apnoea patients with a low arousal threshold. Clin $\mathrm{Sci}$ (Lond). 2011;120(12):505-514.

18. Heinzer RC, White DP, Jordan AS, et al. Trazodone increases arousal threshold in obstructive sleep apnoea.Eur RespirJ. 2008;31(6):1308-1312.

19. Chiu KL, Ryan CM, Shiota S, et al. Fluid shift by lower body positive pressure increases pharyngeal resistance in healthy subjects. Am J Respir Crit Care Med. 2006;174(12):1378-1383.

20. Su MC, Chiu KL, Ruttanaumpawan P, et al. Lower body positive pressure increases upper airway collapsibility in healthy subjects. Respir Physiol Neurobiol. 2008;161(3):306-312.

21. Neelapu BC, Kharbanda OP, Sardana HK, et al. Craniofacial and upper airway morphology in adult obstructive sleep apnea patients: A systematic review and meta-analysis of cephalometric studies. Sleep Med Rev. 2016;31:79-90.

22. Hamoda MM, Kohzuka Y, Almeida FR. Oral Appliances for the Management of OSA-An Updated Review of the Literature, Chest. 2017.

23. Dal Fabbro C, Chaves-Júnior CM, Tufik S. Dentistry in sleep medicine. $1^{\text {st }}$ ed. : Brazil: Dental Press; 2010.
24. Hsu CC, Wu JH, Chiu HC, et al. Evaluating the sleep quality of obstructive sleep apnea patients after continuous positive airway pressure treatment. Comput Biol Med. 2013;43(7):870-878.

25. Spicuzza L, Caruso D, Di Maria G. Obstructive sleep apnoea syndrome and its management. Ther Adv Chronic Dis. 2015;6(5):273-285.

26. Michels D, Rodrigues A, Nakanishi M, et al. Nasal involvement in obstructive sleep apnea syndrome. Int J Otolaryngol. 2014;2014:8.

27. Lee SA, Paek JH, Han SH. Sleep hygiene and its association with daytime sleepiness, depressive symptoms, and quality of life in patients with mild obstructive sleep apnea. J Neurol Sci. 2015;359(1-2):445-449.

28. Ramar K, Dort LC, Katz SG, et al. Clinical Practice Guideline for the Treatment of Obstructive Sleep Apnea and Snoring with Oral Appliance Therapy: An Update for 2015. J Clin Sleep Med. 2015;11(7):773-827.

29. Mostafiz W, Dalci O, Sutherland K, et al. Influence of Oral and Craniofacial Dimensions on Mandibular Advancement Splint Treatment Outcome in Patients with Obstructive Sleep Apnea. Chest. 2015;139(6):1331-1339.

30. Pliska BT, Almeida F. Effectiveness and Outcome of Oral Appliance Therapy. Dent Clin N Am. 2012;56(2):433-444.

31. Dal Fabbro C, Chaves CM. Treatment with intraoral appliances (AIO) In: Dal-Fabbro C, editor, A dentistry in sleep medicine. Maringa, Brazil: Dental Press International; 2009. 201-228 p.

32. Almeida FR, Bittencourt LR, Almeida CIR, et al. Effects of mandibular posture on obstructive sleep apnea severity and temporomandibular joint in patients fitted with an oral appliance. Sleep. 2002;25(5):507-513.

33. Almeida FR, Parker JA, Hodges JS, et al. Effect of a titration polysomnogram on Treatment Success with a mandibular repositioning appliance. J Clin Sleep Med. 2009;5(3):198-204.

34. Tsuiki S, Kobayashi M, Namba K, et al. Optimal positive airway pressure predicts oral appliance response to sleep apnoea. Eur Respir J. 2010;35(5):1098-105.

35. Otsuka R, Almeida FR, Lowe AA, et al. A comparison of responders and nonresponders to oral appliance therapy for the treatment of obstructive sleep apnea. Am J Orthod Dentofacial Orthop. 2006;129(2):222-229. 